\title{
EchoGéo
}

$32 \mid 2015$

Inde : le grand écart spatial

\section{Pauvreté et inégalités en Inde rurale}

Réflexion à partir de deux diagnostics agraires dans l'État du Gujarat

Claire Aubron, Hugo Lehoux et Corentin Lucas

\section{(2) OpenEdition}

Journals

Édition électronique

URL : https://journals.openedition.org/echogeo/14226

DOI : $10.4000 /$ echogeo.14226

ISSN : 1963-1197

Éditeur

Pôle de recherche pour l'organisation et la diffusion de l'information géographique (CNRS UMR 8586)

Référence électronique

Claire Aubron, Hugo Lehoux et Corentin Lucas, "Pauvreté et inégalités en Inde rurale », EchoGéo [En ligne], 32 | 2015, mis en ligne le 15 juillet 2015, consulté le 10 août 2021. URL : http://

journals.openedition.org/echogeo/14226 ; DOI : https://doi.org/10.4000/echogeo.14226

Ce document a été généré automatiquement le 10 août 2021.

EchoGéo est mis à disposition selon les termes de la licence Creative Commons Attribution - Pas d'Utilisation Commerciale - Pas de Modification 4.0 International (CC BY-NC-ND) 


\title{
Pauvreté et inégalités en Inde rurale
}

\author{
Réflexion à partir de deux diagnostics agraires dans l'État du Gujarat
}

\author{
Claire Aubron, Hugo Lehoux et Corentin Lucas
}

\section{NOTE DE L'AUTEUR}

Ce travail a bénéficié du soutien financier d'AgreenSkills (bourse de mobilité), de la région Réunion (projet ARChE_Net) et de l'Agence Nationale de la Recherche (projet EPAD). Nous remercions les agriculteurs des deux cantons pour leur accueil et les relecteurs pour leurs commentaires stimulants. Les propos présentés dans cet article n'engagent que leurs auteurs.

\section{Introduction}

1 Même si la pauvreté urbaine de l'Inde et de ses bidonvilles n'échappe à aucun observateur, c'est en zone rurale, d'après les données statistiques ${ }^{1}$, que vivent la plupart des pauvres du sous-continent : $70 \%$ des Indiens et sensiblement autant des pauvres de l'Inde vivent en zone rurale (Himanshu et al., 2013 ; Planning commission, 2014). L'agriculture, qui constitue l'activité principale de $60 \%$ des actifs ruraux (Himanshu et al., 2013), est indissociable de cette pauvreté rurale et donc du "grand écart de l'Inde " (Shah, 2002 ; Landy, 2010). Elle est parfois décrite comme une activité résiduelle, que les plus riches abandonnent et qui n'implique plus les masses de travailleurs ruraux du passé (Gupta, 2005). Dans un contexte de faible croissance du secteur agricole par rapport au reste de l'économie depuis les années 1990 (Himanshu, 2008), les emplois non agricoles des ruraux sont l'objet d'une attention croissante et suscitent de nouveaux espoirs de sortie de la pauvreté (Nayyar and Sharma, 2005). Le nombre de ces emplois non agricoles à l'échelle de l'Inde est effectivement croissant et des travaux montrent que leur essor a permis de réduire la pauvreté dans les zones étudiées, avec toutefois un renforcement des inégalités (Himanshu et al., 2013). 
2 Autant de questions souvent abordées à l'échelle nationale, qu'il convient d'étudier à l'échelle locale pour faire surgir des facteurs et des processus invisibles à d'autres niveaux d'étude. L'article contribue à cet objectif à travers l'analyse de deux systèmes agraires dans le Gujarat. Situé dans le Nord-Ouest de l'Inde, l'État du Gujarat a connu une forte croissance économique au cours des deux dernières décennies (Bagchi et al., 2005 ; Dixit, 2009), qui lui vaut souvent d'être érigé en modèle à même d'inspirer le reste de l'Inde. Le Gujarat compte plusieurs centres industriels importants et dispose de bonnes infrastructures de transport. Cette croissance concerne les secteurs secondaire et tertiaire, mais aussi - chose plus rare dans l'Inde contemporaine - l'agriculture ${ }^{2}$ (Shah et al., 2009). Dans cet État symbole de l'émergence de l'Inde, une analyse des systèmes agraires et des relations entre agriculture et pauvreté rurale apparaît particulièrement pertinente. La pauvreté rurale existe-t-elle au Gujarat, et si oui quelles sont ses formes? Est-elle homogène ou observe-t-on des inégalités entre les familles à l'intérieur des campagnes? Quelles sont ses origines ? Comment les activités non agricoles - dont les opportunités sont a priori nombreuses du fait de la croissance économique dans les différents secteurs - s'articulent-elles aux activités agricoles et dans quelle mesure ces combinaisons d'activités permettent de résorber la pauvreté ?

Pour répondre à ces questions, nous nous appuyons sur deux travaux de terrain menés d'avril à août 2014 (Lucas, 2014 ; Lehoux, 2014), mobilisant le cadre d'analyse des systèmes agraires proposé par l'agriculture comparée (Cochet et Devienne, 2006; Dufumier, 2007 ; Cochet, 2011). Au cours d'une première étape, nous avons caractérisé le milieu biophysique à partir d'observations et reconstitué l'histoire agraire des dernières décennies par le biais d'entretiens auprès de personnes âgées (entre 20 et 30 dans chaque canton), complétés par la bibliographie. Ce matériau - une compréhension spatialement située des trajectoires différenciées des exploitations agricoles au cours des dernières décennies - nous a permis d'élaborer une première typologie des exploitations agricoles dans chaque canton. Celle-ci nous a servi de base pour définir un échantillonnage permettant ensuite d'analyser plus finement le fonctionnement technique, social et économique de chacun de ces systèmes de production, à partir d'une soixantaine d'entretiens auprès d'agriculteurs dans chaque canton. Nous avons finalement modélisé cette diversité de systèmes de production en construisant des archétypes représentant chacun des ensembles d'exploitations. Les éléments chiffrés présentés dans cet article sont issus de ce travail de modélisation.

\section{Un dense tissu d'exploitations agricoles : genèse et structure actuelle}


Illustration 1 - Carte du Gujarat et localisation des zones d'étude

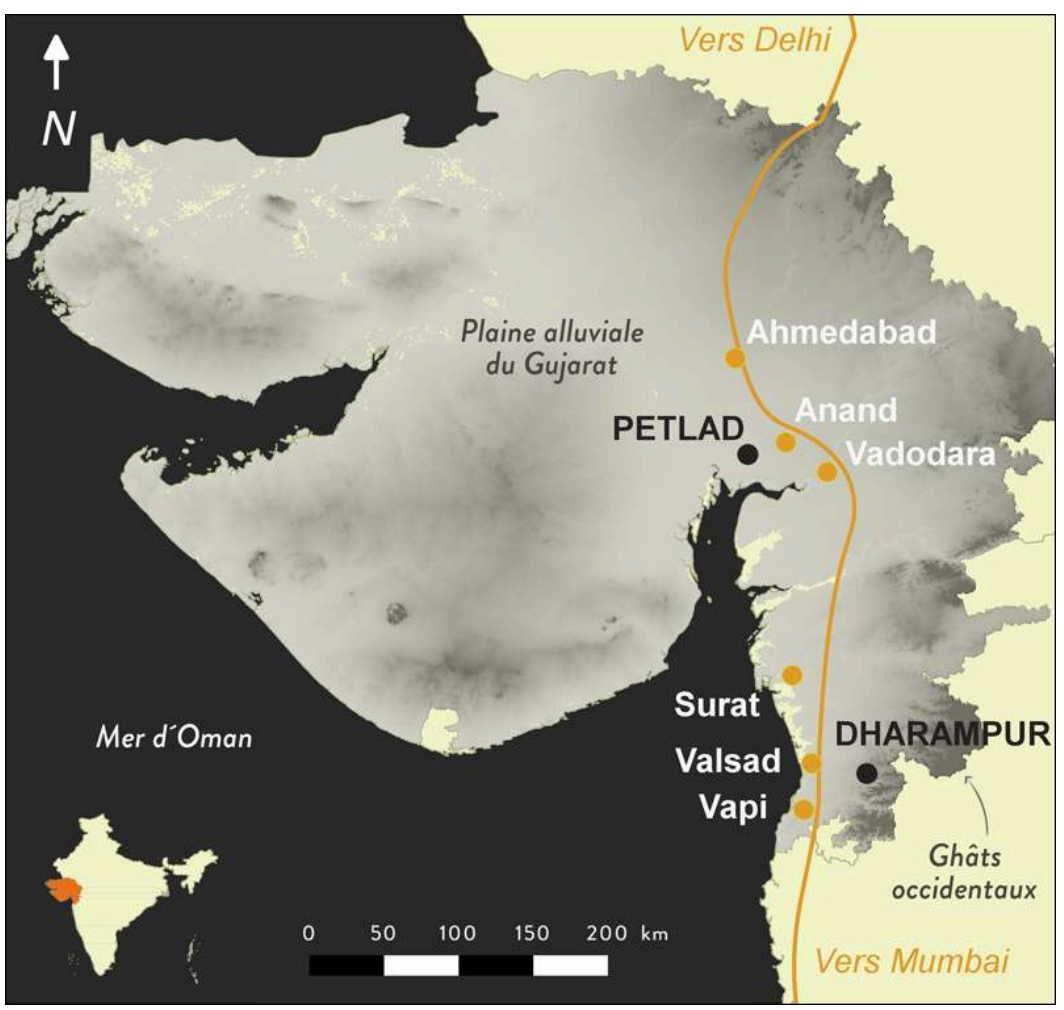

Sources : OpenStreetMap, 2015, OpenStreetMAp et ses contributeurs, http://www.openstreetmap.org/ copyright; réalisation : C. Aubron, H. Lehoux et C. Lucas ; logiciel QGis.

Les deux zones d'étude correspondent à deux espaces de plaine alluviale du Gujarat (voir illustration 1). Le canton de Petlad, au nord, présente très peu de relief et correspond à une plaine aujourd'hui quasi intégralement cultivée et irriguée. Plus au sud, le canton de Dharampur est situé à la frontière entre, à l'ouest, une plaine irriguée donnant sur la mer d'Oman et, à l'est, les montagnes bordant le plateau du Deccan (Ghats occidentaux) aux possibilités d'irrigation réduites. Les deux zones sont soumises au régime des moussons à partir du mois de juin, mais Petlad, qui n'est pas si loin du désert du Kutch marquant la frontière Nord du Gujarat avec le Pakistan, est bien moins arrosé $(800 \mathrm{~mm}$ de précipitations annuelles) que Dharampur $(2500 \mathrm{~mm})$. Les deux cantons sont aujourd'hui organisés autour d'une petite ville, qui rassemble 60000 habitants dans le cas de Petlad et 30000 à Dharampur, et sont situées à proximité du principal axe routier et ferroviaire joignant Delhi à Mumbai. Les centres industriels de Vadodara et d'Ahmedabad, assez anciens et à la croissance économique aujourd'hui ralentie, sont à moins de $70 \mathrm{~km}$ de Petlad. Dharampur est très proche de Surat et Vapi, dont les activités industrielles sont en pleine expansion, notamment dans le secteur pétrochimique.

\section{Une mise en valeur agricole ancienne}

Caractéristique du Nord de l'Inde, l'agriculture de Petlad a longtemps eu le mil pour pivot, associé à des légumineuses, auxquels s'ajoutait du riz cultivé dans les bas-fonds, tout cela en période de mousson. Les castes qui contrôlaient la région $\mathrm{du} \mathrm{V}^{\mathrm{e}} \mathrm{au}$ $\mathrm{XIII}^{\mathrm{e}}$ siècle (les Solanki, Parmar et Chauhan) auraient progressivement perdu ce pouvoir 
à partir du Sultanat de Delhi, puis de l'Empire moghol, au profit de la caste des Patel (Deumari, 2011). Ces derniers, qui seraient originaires du Punjab depuis lequel ils auraient migré vers cette partie du Gujarat entre 500 et 1000 , sont devenus collecteurs des impôts pour le compte de l'Empire moghol au XVII ${ }^{e}$ siècle, puis de la couronne britannique (Randhawa, 1980). Ils ont ainsi acquis ou conforté des droits de propriété foncière, qui se sont progressivement étendus à presque toute la zone. Ils sont à l'origine des premières cultures irriguées, notamment le tabac, à proximité des puits qu'ils ont fait aménager (Shah et Shah, 1950). En 1950, ils disposent ainsi de grandes exploitations de 3 à 20 ha cultivés, qui sont travaillées par des employés permanents ${ }^{3}$ et des journaliers issus du reste de la population (castes des Solanki, Parmar et Chauhan, auxquelles sont venus s'ajouter les Thakor et les Vagri, probablement suite à des migrations). L'élevage joue alors un rôle majeur pour renouveler la fertilité des sols cultivés et comme force de traction pour le travail du sol et le transport. C'est une caste d'éleveurs, les Rabhari, rejointe plus tard par les Bharvar, qui, en 1950, était chargée de conduire les animaux au pâturage et possèdait elle-même des troupeaux de ruminants.

Pendant longtemps, les seuls habitants de la zone Sud (Dharampur) ont été des Adivasis $^{4}$ ("tribaux »), qui occupaient le sous-continent indien avant l'arrivée des Aryens. Les Adivasis pratiquaient la chasse et la cueillette dans la forêt qui constituait à l'époque leur environnement. Ils maîtrisaient également l'abattis-brûlis et l'agroforesterie avec brûlis de feuilles qui leur permettaient de cultiver du riz ainsi que de l'éleusine, du sorgho et du mil. C'est au XIII ${ }^{e}$ siècle qu'une famille de Rajputs, originaires du Rajasthan, qui combattaient les musulmans du Sultanat de Delhi, installa un petit royaume à Dharampur (Administration of Dharampur, 1921). Tout en collectant les impôts pour les Moghols puis les Britanniques, celui-ci fonctionna de manière relativement autonome jusqu'à l'indépendance de l'Inde. En échange de services rendus, la famille du roi distribua des terres dans la plaine à des parents Rajputs, ainsi qu'à des familles des castes Brahmanes, Parsi, Soni et Patel, à hauteur de 20 à 100 ha par propriété. Après défrichement, ces espaces furent cultivés chaque année avec du riz et des légumineuses, en période de mousson. Des Adivasis employés permanents ou métayers ${ }^{5}$ fournissaient l'essentiel de la main-d'œuvre dans ces propriétés de la plaine, pendant que les autres pratiquaient des cultures de mousson dans les montagnes pour leur propre compte. Dans la plaine, l'élevage avait le même rôle crucial qu'à Petlad. Les transferts de fertilité étaient là aussi assurés par une caste de pasteurs, les Ahir, éleveurs de grands troupeaux bovins et caprins.

\section{Réforme agraire, révolutions verte et blanche}

7 Comme dans d'autres régions de l'Inde, les lois de réforme agraire des années suivant l'indépendance ont modifié la répartition du foncier dans les deux cantons, sans toutefois la remettre fondamentalement en cause. L'absence de réelle volonté politique, notamment de la part des États qui comptaient souvent les élites foncières locales parmi leurs alliés, et les exemptions prévues par les lois ou rendues possibles par l'ambiguïté des textes expliquent que le processus de réforme agraire indien ait été inabouti (Appu, 1997 ; Pouchepadass, 2006 ; Breman, 2007a). À Petlad, certains Solanki, Parmar, Chauhan et Thakor qui travaillaient sur les propriétés des Patel ont accédé à de petits lopins de terre d'un à deux hectares, rejoignant le lot des quelques petits propriétaires qui existaient déjà dans la région. Ce sont néanmoins les terres les moins fertiles qui ont été ainsi redistribuées. Les surfaces concernées, faibles à l'origine, le 
sont devenues plus encore du fait des transactions foncières ultérieures. En effet, certains bénéficiaires de la réforme agraire ont ensuite cédé les terres reçues aux Patel, soit dans le cadre de vente volontaire, soit dans le cadre de crédit-bail qu'ils ne parvenaient pas à rembourser. Ce mécanisme de transferts de droits pour endettement, qui est évoqué sous une forme temporaire par Servet (2007), est encore en vigueur aujourd'hui. Mais surtout, la grande majorité des travailleurs agricoles de même que les pasteurs n'ont pas bénéficié de la réforme agraire et sont restés sans terre.

8 À Dharampur, la plupart des grandes propriétés de la plaine accordées dans le passé par le roi ont vu leur taille diminuer, mais certaines d'entre elles n'ont pas du tout été affectées. Certains employés permanents adivasis et éleveurs caprins Ahir ont accédé au foncier directement ou par achat après la réforme agraire, constituant des exploitations familiales de 0,5 à 4 ha dans la plaine. Les autres Adivasis sont devenus formellement propriétaires des terres qu'ils cultivaient dans les montagnes. À la différence de Petlad, il n'y a donc pas d'importante population de paysans sans terre à Dharampur. L'autre facteur d'évolution de la structure foncière dans les deux zones est la division des terres en propriété entre fils ${ }^{6}$, à chaque génération.

9 À partir des années 1970, la révolution verte et ses trois ingrédients - irrigation ${ }^{7}$, engrais chimiques et variétés à haut potentiel de rendement - (Dorin et Landy, 2002) ont transformé l'agriculture de la plaine dans les deux cantons. Dans celui de Petlad, les parcelles accueillent généralement deux cycles de culture par an, et parfois même trois. Les systèmes de culture se sont réorganisés autour du tabac, culture irriguée d'hiver exigeante en travail mais très rémunératrice. Dans la plaine de Dharampur, la double culture annuelle riz / sorgho est la règle dans les petites et moyennes exploitations. Les plus grandes se sont tournées vers des cultures moins intensives en travail et plus rémunératrices : la canne à sucre, et à partir des années 1990 les vergers de mangues. Les toutes petites exploitations (moins d'un ha) formées par les divisions successives ont, au cours des années 2000, développé du maraîchage, dont les produits sont prisés sur le marché émergent de la petite ville de Dharampur. Avec le développement de la collecte laitière et de la fourniture d'intrants d'élevage à bas prix (insémination artificielle, concentrés) par les coopératives laitières liées à AMUL ${ }^{8}$, presque tous les agriculteurs de la plaine dans les deux zones sont devenus des éleveurs laitiers, y compris ceux qui n'avaient pas de terre. Certains propriétaires Patel de Petlad et une partie des anciens éleveurs caprins Ahir de Dharampur ont investi dans de plus grands élevages laitiers, rassemblant de 30 à 200 mères. Même si ce point ne sera pas développé ici, notons que ces évolutions posent des questions environnementales tant locales (quantité et qualité de l'eau) que globales (consommation d'énergie fossile, émissions de gaz à effet de serre).

Les montagnes de Dharampur, du fait de possibilités d'irrigation réduites, sont restées à l'écart de ces révolutions verte et blanche. Pour subvenir aux besoins d'une population croissante, les agriculteurs adivasis ont étendu les zones de culture de mousson près des maisons (riz et légumineuses) comme plus éloignées (éleusine) sur la forêt. Le gouvernement, qui aurait dans un premier temps contribué à cette déforestation par des prélèvements de bois, a ensuite délimité des espaces de reboisement, sur lesquels les activités agricoles sont théoriquement interdites. La déforestation a réduit la biomasse produite dans les forêts qui jouait jusque-là un rôle central dans le renouvellement de la fertilité des terres cultivées. Cela n'est sans doute pas sans rapport avec la baisse des rendements évoquée par les agriculteurs et les faibles valeurs 
issues de nos observations et enquêtes ( 0,5 à $1,2 \mathrm{t} /$ ha pour le riz en montagne contre 2 à $3,5 \mathrm{t} / \mathrm{ha}$ dans la plaine voisine). Notons que certaines familles adivasis en montagne ont pu accéder à l'irrigation, via l'installation de pompes dans les cours d'eau et ont, elles aussi, développé une activité marâichère. Ces investissements ont été favorisés par des prêts et des subventions pour équipement accordés par des programmes d'État ayant pour cible les populations tribales.

Tableau 1 - Caractéristiques des systèmes de production de la zone de Petlad

\begin{tabular}{|c|c|c|c|c|}
\hline Type & Main-d'œuvre & Surface (ha) & $\begin{array}{l}\text { Systèmes de } \\
\text { culture }\end{array}$ & $\begin{array}{l}\text { Systèmes } \\
\text { d'élevage }\end{array}$ \\
\hline $\begin{array}{l}\text { Propriétaires confiant } \\
\text { les terres en } \\
\text { métayage }\end{array}$ & Métayers & $0,2-9$ & $\begin{array}{l}\text { Tabac/mil ; Tabac; } \\
\text { Tabac//banane; } \\
\text { Tabac/riz }\end{array}$ & $\begin{array}{l}\text { 0-1 vache croisée } \\
\text { Holstein ou Jersey }\end{array}$ \\
\hline $\begin{array}{l}\text { Propriétaires } \\
\text { éleveurs laitiers }\end{array}$ & $\begin{array}{l}\text { Familiale (et } \\
\text { salariés } \\
\text { permanents) }\end{array}$ & $0,9-2$ & $\begin{array}{l}\text { Luzerne/sorgho ; } \\
\text { Maïs/sorgho }\end{array}$ & $\begin{array}{l}12-50 \text { vaches } \\
\text { croisées }\end{array}$ \\
\hline $\begin{array}{l}\text { Propriétaires aux } \\
\text { productions } \\
\text { diversifiées }\end{array}$ & $\begin{array}{l}\text { Familiale et } \\
\text { salariés } \\
\text { journaliers }\end{array}$ & $0,4-2$ & $\begin{array}{l}\text { Tabac/riz; Tabac/ } \\
\text { mil ; Herbe à } \\
\text { éléphant }\end{array}$ & $\begin{array}{l}\text { 4-12 vaches } \\
\text { croisées }\end{array}$ \\
\hline Petits propriétaires & $\begin{array}{l}\text { Familiale et } \\
\text { salariés } \\
\text { journaliers }\end{array}$ & $0,1-0,36$ & $\begin{array}{l}\text { Tabac/riz; Tabac/ } \\
\text { mil }\end{array}$ & 1-2 bufflesses \\
\hline Métayers & $\begin{array}{l}\text { Familiale et } \\
\text { salariés } \\
\text { journaliers }\end{array}$ & $\begin{array}{l}0 \quad(0,5-3 \text { en } \\
\text { métayage })\end{array}$ & $\begin{array}{l}\text { Tabac/mil ; Tabac; } \\
\text { Tabac//banane; } \\
\text { Tabac/riz }\end{array}$ & 0-2 bufflesses \\
\hline Journaliers & Familiale & 0 & - & $0-1$ bufflesse \\
\hline Pasteurs & Familiale & 0 & - & $\begin{array}{l}5-25 \text { buffles et } \\
\text { bovins de race } \\
\text { locale }\end{array}$ \\
\hline
\end{tabular}

Dans un village du canton, nous avons estimé l'importance relative des systèmes de production en nombre de familles à partir des données de livraison du lait à la coopérative. La répartition obtenue est la suivante : propriétaires confiant leurs terres en métayage : 2 à $5 \%$; propriétaires éleveurs laitiers : $<0,1 \%$; propriétaires aux productions diversifiées : 2 à $15 \%$; petits propriétaires : 50 à $70 \%$; métayers et journaliers : 15 à $30 \%$; Pasteurs : 1 à $5 \%$.

Dans le district d'Anand auquel appartient le canton de Petlad, d'après les données du recensement 2011 , il y a 287000 « ouvriers agricoles » (qui englobent nos deux catégories métayers et journaliers), et 204000 « cultivateurs » (des propriétaires ou des locataires de terre travaillant plus de 6 mois par an dans l'agriculture).

La simple barre sépare les cultures d'une rotation annuelle (Tabac/mil : le tabac et le mil sont cultivés I'un après l'autre sur la parcelle la même année) et la double barre celles de rotations pluriannuelles (Tabac//banane : une année avec seulement du tabac cultivé puis plantation de banane l'année suivante). 
Tableau 2 - Caractéristiques des systèmes de production de la zone de Dharampur

\begin{tabular}{|c|c|c|c|c|c|}
\hline Type & Main-d'œuvre & $\begin{array}{l}\text { Surface } \\
\text { (ha) }\end{array}$ & $\begin{array}{l}\text { Accès } \\
\text { à l'eau }\end{array}$ & $\begin{array}{l}\text { Systèmes } \\
\text { culture }\end{array}$ & $\begin{array}{l}\text { Systèmes } \\
\text { d'élevage }\end{array}$ \\
\hline $\begin{array}{l}\text { Producteurs de } \\
\text { mangues }\end{array}$ & $\begin{array}{l}\text { Salariés } \\
\text { permanents et } \\
\text { temporaires }\end{array}$ & $4-40$ & Oui & $\begin{array}{l}\text { Manguiers irrigués; } \\
\text { Teck }\end{array}$ & - \\
\hline $\begin{array}{l}\text { Agriculteurs } \\
\text { pluriactifs à } \\
\text { canne à sucre }\end{array}$ & $\begin{array}{l}\text { Familiale et } \\
\text { salariés } \\
\text { temporaires }\end{array}$ & $<4$ & Oui & $\begin{array}{l}\text { Canne }_{2} / / \text { riz } \\
\text { Manguiers irrigués }\end{array}$ & - \\
\hline Eleveurs laitiers & $\begin{array}{l}\text { Familiale et } \\
\text { salariés } \\
\text { permanents }\end{array}$ & $<4$ & Oui & $\begin{array}{l}\text { Riz/sorgho; } \\
\text { Manguiers non } \\
\text { irrigués }\end{array}$ & $\begin{array}{l}\text { 15-100 bufflesses ou } \\
\text { vaches croisées }\end{array}$ \\
\hline $\begin{array}{l}\text { Agriculteurs aux } \\
\text { productions } \\
\text { diversifiées }\end{array}$ & Familiale & $<4$ & Oui & $\begin{array}{l}\text { Riz/sorgho; } \\
\text { Canne }_{2} / / \text { riz; } \\
\text { Manguiers non } \\
\text { irrigués }\end{array}$ & $\begin{array}{l}1-5 \text { bufflesses ou } \\
\text { vaches croisées }\end{array}$ \\
\hline Maraîchers & $\begin{array}{l}\text { Familiale et } \\
\text { salariés } \\
\text { permanents }\end{array}$ & $<1$ & Oui & Légumes divers & - \\
\hline Pasteurs & Familiale & $<2$ & Non & Riz ; Légumineuses & 20-100 chèvres \\
\hline $\begin{array}{l}\text { Agriculteurs en } \\
\text { culture pluviale }\end{array}$ & Familiale & $<2$ & Non & $\begin{array}{l}\text { Riz; Légumineuses; } \\
\text { Eleusine }\end{array}$ & $\begin{array}{l}0-2 \text { bovins de race } \\
\text { locale }\end{array}$ \\
\hline
\end{tabular}

Nous estimons qu'il y a dans le canton 15 familles spécialisées dans la production de mangue et 30 à 40 spécialisées dans l'élevage laitier. En 2008, le Gouvernement du Gujarat dénombrait 165662 Adivasis (Scheduled tribes) dans le canton de Dharampur, soit $91 \%$ de la population (Patel, 2011). La majorité d'entre eux travaille probablement dans l'agriculture $-84 \%$ de la population active du canton travaille dans l'agriculture d'après le recensement de 2011, mais tous ne sont pas des « agriculteurs en culture pluviale $»$.

Canne2 signifie que la canne à sucre reste en culture pendant 2 ans.

\section{Une myriade d'exploitations liées par des flux de main-d'œuvre}

Les exploitations agricoles, du fait de leur petite taille et de leur occupation quasi continue de l'espace dans la plaine irriguée, forment un tissu particulièrement dense dans les deux cantons. Cette densité liée à la structure foncière se double d'une densité «sociale » générée par les flux de main-d'œuvre entre exploitations. Presque tous les propriétaires en zone irriguée - les producteurs de mangue, les agriculteurs pluriactifs, les éleveurs laitiers, les maraîchers à Dharampur et les familles ayant plus de 0,1 ha à Petlad - font en effet appel à de la main-d'œuvre extérieure à la famille, pour conduire tout ou partie de leurs cultures comme de leurs activités d'élevage (voir tableaux 1 et 2). D'après nos calculs, cette main-d'œuvre extérieure réalise jusqu'à $95 \%$ du travail dans certaines catégories d'exploitations, ce qui constitue un indicateur de l'échec d'une réforme agraire qui entendait donner la terre à ceux qui la travaillent. Elle est 
fournie par des métayers ${ }^{9}$ (pour les cultures, à Petlad), des salariés journaliers (pour les cultures, dans les deux cantons), des salariés à la saison ou à la tâche (pour les cultures, à Dharampur) ou des salariés permanents (pour l'élevage, dans les deux cantons). Ces travailleurs peuvent être avec ou sans terres, qu'ils cultivent pour leur propre compte : c'est le cas de certains métayers de Petlad qui ont reçu des terres à la réforme agraire et des cultivateurs adivasis de la montagne à Dharampur, qui viennent faire le repiquage du riz ou la récolte des mangues dans la plaine. Dans un certain nombre de cas, ces travailleurs élèvent aussi des animaux laitiers pour leur propre compte, pour l'autoconsommation et pour la vente. Les différents types de travailleurs peuvent être combinés dans une exploitation, le propriétaire faisant appel à eux en fonction des tâches à réaliser. À Petlad, les métayers emploient généralement des journaliers pour la récolte du tabac.

Outre ces flux de main-d'œuvre, des transferts temporaires de droits fonciers entre exploitations existent. Au métayage déjà évoqué à Petlad, s'ajoute la location de terres qui s'est développée avec l'essor des migrations internationales de certaines familles Patel, à partir des années 1970 : les propriétaires absents qui vivent aux États-Unis, au Royaume-Uni ou au Canada louent leurs terres à des parents, qui les font ensuite généralement travailler par des métayers ou des salariés. Sur une partie des terres irriguées de Petlad, rapports fonciers et division sociale du travail dessinent ainsi un système à quatre étages impliquant le propriétaire absent, le propriétaire présent confiant ses terres en métayage, les métayers, et les journaliers embauchés par les métayers. La figure de l'illustration 4 met en regard la contribution au travail de ces différentes catégories sociales et la part de la valeur ajoutée qu'elles récupèrent.

\section{Pauvreté du plus grand nombre et pouvoir économique des propriétaires fonciers}

\section{Paysans sans terre et paysans sans eau dans l'extrême pauvreté}

13 Les figures des illustrations 2 et 3 font apparaître les revenus issus de l'agriculture par système de production. Concentrons-nous d'abord sur la partie inférieure du graphique, qui correspond à des catégories sociales qui sont loin d'être numériquement négligeables. Dans les deux zones, nous estimons en effet qu'au moins $30 \%$ des familles tirent de l'agriculture un revenu ${ }^{10}$ qui est inférieur au seuil de pauvreté national récemment redéfini ( 32 roupies soit $0,41 € /$ personne/jour au moment de l'étude, d'après Planning Commision, 2014 $4^{11}$ ) (voir illustrations 2 et 3). Si ce résultat était attendu pour le canton de Dharampur, considéré comme pauvre parce que " tribal ", cela n'était en revanche pas le cas pour le canton de Petlad. Certes densément peuplée, la zone de Petlad est en effet une plaine fertile où les révolutions verte et blanche (cette dernière ayant là son berceau), ont permis une forte croissance agricole. C'est l'accès à la terre en propriété à Petlad et à l'eau d'irrigation à Dharampur qui conditionnent l'obtention d'un revenu agricole supérieur au seuil de pauvreté national (voir illustrations 2 et 3). On retrouve là un constat établi depuis longtemps en Inde rurale, où les plus pauvres sont invariablement ceux qui n'ont pas de terres ou pas d'eau pour les irriguer (Shah, 2005 ; Pouchepadass, 2006); mais le nombre de foyers concernés, dans un État érigé en modèle de "l'émergence », est impressionnant. 
La situation est d'autant plus préoccupante que certaines des évolutions en cours contribuent à l'aggraver. Si elle est avérée, la crise de la fertilité des sols qui affecte l'agriculture des montagnes de Dharampur, fera ainsi diminuer les revenus agricoles des familles adivasis qui n'ont que des cultures pluviales. Ces derniers seront d'autant moins en capacité de la résoudre, qu'ils n'ont pas les moyens d'acheter des engrais et qu'ils utilisent une partie des déjections animales comme source d'énergie pour la cuisine, et des pailles de riz pour payer la mouture des grains. Cela suggère que l'accès à la biomasse peut être tout aussi crucial que l'accès aux terres de culture : s'il est difficile de vivre de l'agriculture avec une terre sans eau dans de nombreuses régions de l'Inde, qu'en est-il quand, de plus, on ne dispose plus des moyens pour renouveler sa fertilité ? La division des terres entre les fils lors de la transmission des plus petites propriétés est un autre facteur de paupérisation des agriculteurs. Ce processus continue de se produire en moyenne à l'échelle de l'Inde (Dorin et al., 2013) et dans les deux zones étudiées. Il fait basculer des petits propriétaires dans la catégorie des micropropriétaires, dont les revenus agricoles sont moindres sauf s'ils parviennent à développer du maraîchage ou de l'élevage laitier. Certains l'ont fait avec succès dans les deux cantons, mais cela suppose de disposer de terres irrigables. Les prêts ou les subventions accordés par les coopératives laitières et l'État pour l'acquisition des équipements d'irrigation et des animaux d'élevage semblent par ailleurs jouer un rôle déterminant dans ces trajectoires. En deçà de 0,08 ha par fils en propriété, les familles de Petlad ne divisent pas leurs terres: elles les transmettent à l'un d'entre eux seulement, les autres fils rejoignant le rang des sans-terres. Enfin, la diminution des ressources en eau et l'augmentation du coût de leur prélèvement du fait de l'abaissement du niveau des nappes, contribuent à faire basculer des agriculteurs ayant accès à l'irrigation dans la catégorie des agriculteurs sans eau. Observé à Dharampur, ce processus est en cours dans certaines régions de l'Inde (Shah, 2009), et a été décrit dans le Nord du Gujarat (Barry et Issoufaly, 2006). Il concourt à renforcer les inégalités, seuls les plus riches propriétaires étant en mesure d'investir dans des forages toujours plus profonds.

Illustration 2 - Revenu agricole annuel par système de production dans la zone de Petlad

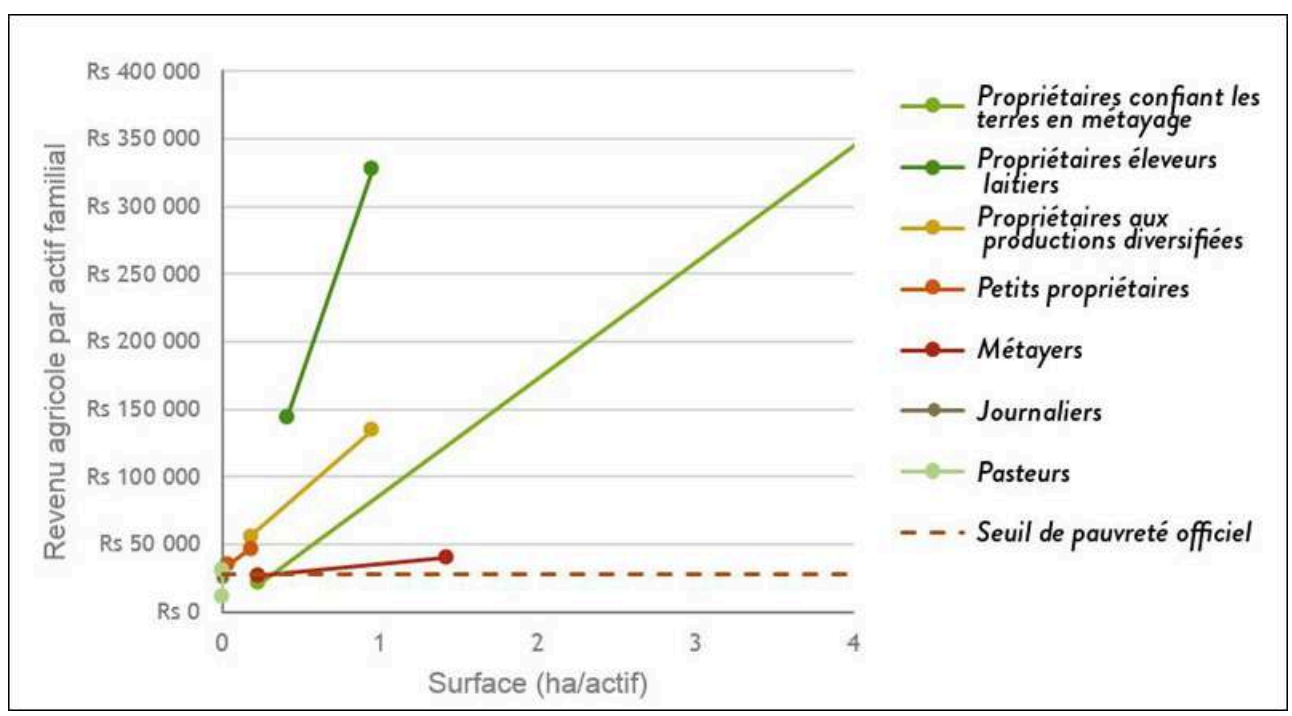


Illustration 3 - Revenu agricole annuel par système de production dans la zone de Dharampur

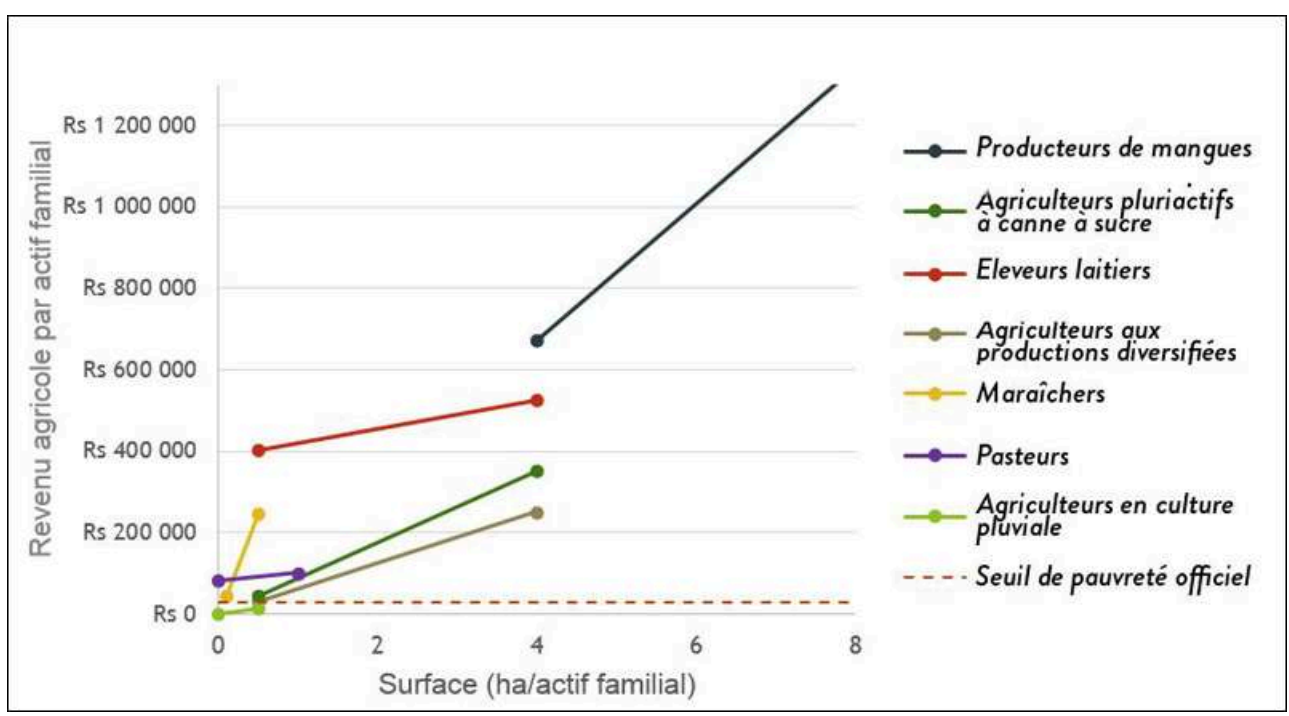

\section{Relations sociales et inégale répartition de la valeur ajoutée}

Mais la pauvreté d'une partie des agriculteurs, dans les deux cantons, n'est pas qu'un problème structurel de faible disponibilité des ressources en terre, en eau ou même en biomasse par famille. Elle est aussi liée aux relations sociales en vigueur et aux modalités de répartition de la valeur ajoutée entre les propriétaires et les métayers ou salariés. Même si nos observations à ce sujet ont été limitées et gagneraient à être complétées, il semble bien que se soit produit au cours des deux dernières décennies une certaine émancipation des catégories sociales défavorisées, semblable à celle décrite dans d'autres parties de l'Inde rurale (Harriss et al., 2012 ; Jodhka, 2014). À la faveur de la fragmentation des grandes propriétés agricoles, du départ d'une partie des propriétaires (Breman, 2007b), du système de quotas par caste dans l'enseignement supérieur et l'administration, du poids politique croissant de certaines catégories d'agriculteurs (Pouchepadass, 2006; Jaffrelot, 2006; Jodhka, 2014), des subventions pour équipements accordées aux plus pauvres ou de leur implication dans des activités non agricoles, une partie des familles des deux zones étudiées est devenue socialement moins dépendantes des propriétaires fonciers. Certains jeunes sans terre de Petlad choisissent ainsi de ne pas prendre de terres en métayage, alors même qu'ils y auraient accès, afin de profiter librement des opportunités qui s'offrent à eux sur le marché du travail journalier - agricole ou pas. 
Illustration 4 - Contribution en travail et partage de la valeur ajoutée nette dans le système Propriétaires-Métayers-Journaliers à Petlad

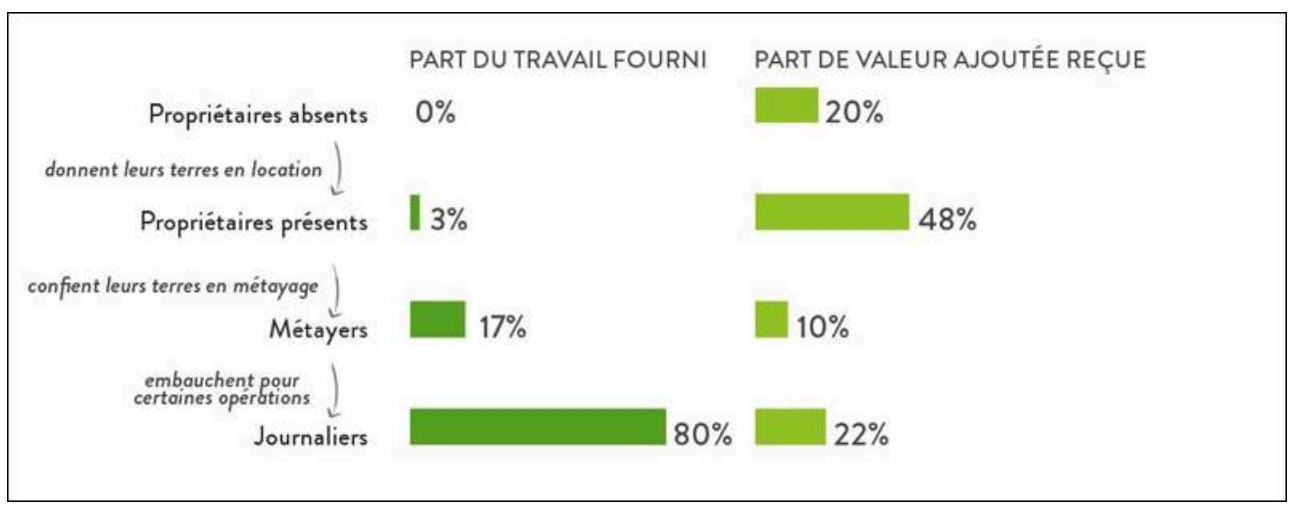

17 Pour autant, les relations entre propriétaires et salariés ou métayers sont loin d'être équilibrées. En témoignent la perpétuation du système de crédit-bail à Petlad, qui dépossède les agriculteurs de leurs terres, les logements de fortune des travailleurs adivasis qui viennent pour quelques semaines dans la plaine, ou encore le travail des enfants dans les vergers de manguiers. Et surtout, la valeur ajoutée de l'agriculture est très inégalement répartie. L'évaluation de la productivité journalière du travail ${ }^{12}$ pour les différents systèmes de culture est à ce titre éclairante : si l'on exclut de l'analyse les systèmes de culture de mousson des montagnes de Dharampur caractérisés par une très faible productivité du travail, on constate que la rémunération des travailleurs journaliers (de 100 à 150 Rs, soit 1,20 à $2 € /$ jour au moment de l'étude) ne correspond au plus qu'à la moitié de la richesse créée par jour de travail - le plus souvent entre un tiers et un cinquième. Dans certains systèmes de culture incluant du tabac ou du piment à Petlad ou pour les vergers de mangue à Dharampur, le salaire journalier ne s'élève qu'à un tiers à un cinquième de la valeur ajoutée, le reste revenant au propriétaire $^{13}$.

Dans les conditions actuelles, les accords de métayage à Petlad semblent plus avantageux pour les travailleurs que le salariat : dans le système détaillé dans la figure de l'illustration 4, l'écart entre la contribution en travail des métayers (17\%) et la part de la valeur ajoutée qu'ils récupèrent $(10 \%)$ est moindre que pour les salariés (qui fournissent $80 \%$ du travail et récupèrent $22 \%$ de la valeur ajoutée). Les accords de métayage sont d'autant plus intéressants qu'ils incluent souvent d'autres avantages non comptabilisés dans ces calculs, tels que des prêts accordés par le propriétaire pour couvrir des frais de santé ou le mariage d'une fille, ou encore le droit pour le métayer de cultiver des fourrages sur le bord des champs. Ces avantages sont accordés dans le cadre de relations entre les familles de propriétaires et de métayers qui durent généralement depuis plusieurs générations. N'est pas métayer qui veut: la caste des Vagri par exemple, accède difficilement à ce statut et est souvent cantonnée au travail salarié. Même si la place du crédit dans ces relations demanderait à être analysée plus finement, elles ne sont pas sans rappeler certaines formes anciennes de paternalisme, voire de travail asservi de l'Inde rurale (Pouchepadass, 2005 ; Breman, 2007a ; Breman et Guérin, 2009). Pour compléter cette comparaison entre métayage et salariat, notons que le métayage s'accompagne d'une plus grande prise de risque et ne permet pas, à la différence du salariat, de disposer immédiatement d'un revenu. Que ce soit dans le cadre du salariat ou du métayage, les propriétaires récupèrent quoiqu'il en soit entre la moitié et les quatre cinquièmes de la valeur ajoutée pour les cultures, pour un travail 
fourni souvent minime (voir illustration 4). Dans les deux zones étudiées, la pauvreté est donc indissociable non seulement de l'inégale répartition de la terre et de l'eau mais aussi de celle de la valeur ajoutée. Cela rejoint les résultats de recherches conduites dans d'autres régions de l'Inde mettant en avant la très faible rémunération du travail dans divers accords de location de terre, de métayage ou de salariat agricole (Rawal, 2006 ; Ramachandran et al., 2010).

\section{Le pouvoir économique des propriétaires fonciers}

19 Si les propriétaires ne sont plus les seuls prescripteurs et qu'un pan de la vie sociale échappe aujourd'hui à leur contrôle, leur pouvoir économique - avec des revenus issus de l'agriculture qui sont 10 à 40 fois supérieurs à ceux obtenus par les plus pauvres et qui atteignent 1,2 millions de roupies ${ }^{14}$ annuels pour certains producteurs de mangue de Dharampur (voir illustrations 2 et 3) - reste fort et déterminant pour l'avenir dans les deux zones. Les plus aisés d'entre eux sont généralement impliqués dans des activités non agricoles localement, ailleurs dans l'État, ou à l'étranger. La part de leur revenu qui provient de l'agriculture est peut-être devenue anecdotique mais ils n'abandonnent pas pour autant leurs terres. Ils les font mettre en culture via différents systèmes impliquant plus ou moins d'intermédiaires, avec, comme évoqué ci-dessus, un partage de la valeur ajoutée qui leur est très favorable. Comme analysé ailleurs au Gujarat par Breman (2007b), c'est bien cette inégale répartition de la terre, de l'eau et de la valeur ajoutée qui leur a permis, et qui permet encore à ceux d'entre eux ayant conservé assez de terres, de capitaliser en réalisant différents investissements. Ces investissements portent sur l'agriculture (infrastructures d'irrigation et en particulier forages, qui à Dharampur sont de plus en plus profonds et donc de plus en plus coûteux ; implantation de vergers de mangues), les activités non agricoles (entreprises de Petlad), les études supérieures des enfants leur ouvrant ensuite l'accès à des emplois non agricoles bien rémunérés ou la migration internationale depuis Petlad.

De l'autre côté de l'échelle sociale, les journaliers et métayers de Petlad et les Adivasis des montagnes de Dharampur sont complètement dépendants des propriétaires pour accéder à des emplois agricoles qui leur sont vitaux. À ce titre, les choix de culture et de modes de conduite que font les propriétaires sont déterminants. À partir des années 1970, le développement de la culture du tabac et la multiplication des cycles de culture avec l'expansion de l'irrigation dans la zone de Petlad ont généré une forte demande en main-d'œuvre, en dépit de l'essor de la moto-mécanisation pour le travail du sol. Cela a permis d'absorber la croissance démographique des métayers et des journaliers, sans toutefois les faire sortir de la pauvreté. Certains propriétaires de Petlad choisissent aujourd'hui de ne faire qu'un cycle de culture par an, le tabac, ou de remplacer le tabac par des plantations d'eucalyptus. À Petlad, de grandes exploitations laitières de plus de 100 vaches interrompent leur activité. A Dharampur, les vergers de manguiers prennent la place du riz et de la canne à sucre dans les plus grandes propriétés. Comme l'ont remarqué plusieurs auteurs (Gadgil et Guha, 1993 ; Breman, 2007a), ce sont autant de changements qui font diminuer la demande en travail agricole - et pour certains accentuent sa saisonnalité - et sont lourds de conséquence pour les plus pauvres. Ils sont motivés par les prix de vente intéressants de ces produits sur le marché (tabac, mangues) et par la hausse des coûts. Les propriétaires avancent notamment que les salaires des journaliers agricoles locaux s'accroissent. C'est ainsi qu'ils justifient également l'acquisition d'équipements motomécanisés qui diminuent le besoin en 
travail. Une autre réponse est le recours à de la main-d'œuvre originaire d'autres régions de l'Inde, probablement encore plus pauvre et donc prête à travailler pour des salaires inférieurs. Les ouvriers agricoles employés dans les grands élevages laitiers de Petlad de même qu'une partie des récolteurs de mangues à Dharampur sont ainsi des migrants de l'Uttar Pradesh et du Bihar ${ }^{15}$. L'offre de travail agricole se voit donc façonnée par les choix des propriétaires fonciers, dans les mains desquels repose finalement le développement agricole des deux zones.

\section{Des activités non agricoles qui ne modifient pas les contours des inégalités}

Que ce soit en haut ou en bas de l'échelle sociale, nombreux sont les agriculteurs des deux régions étudiées qui exercent des activités non agricoles. Seuls les agriculteurs des exploitations travaillant principalement avec de la main-d'œuvre familiale avec un minimum de terres en propriété et d'accès à l'eau sont spécialisés dans l'agriculture. C'est le cas aussi de certains agriculteurs aux revenus agricoles plus faibles mais qui sont plus âgés, et ont donc moins de besoins. Comme dans d'autres régions de l'Inde (Abraham, 2009 ; Himanshu et al., 2013), ces activités non agricoles semblent avoir pris de l'ampleur dans les deux cantons au cours des dernières décennies, notamment pour les plus pauvres. Les familles saisissent l'opportunité que représentent l'augmentation des emplois liés à l'industrialisation et à l'urbanisation et le développement des services, notamment dans les petites villes.

Il est certain qu'il y a là un facteur de changement social (Gupta, 2005 ; Jodhka, 2014) : ces familles sont moins dépendantes des propriétaires fonciers, certains de leurs membres se déplacent fréquemment, découvrent la ville, contribuent à définir ses codes, travaillent dans de nouveaux secteurs, etc. Combiné avec la mise en place d'actions publiques - programme de distribution alimentaire, électrification, amélioration du réseau routier, développement des infrastructures et services de santé -, cet essor des activités non agricoles donne l'image d'une amélioration des conditions de vie et d'une «modernisation" de la société. Qu'en est-il dans les deux cantons étudiés ? Les activités non agricoles permettent-elles de résorber la pauvreté et de réduire les inégalités ? Assiste-t-on à un abandon de l'agriculture par les plus pauvres et à un transfert de main-d'œuvre depuis l'agriculture vers les autres secteurs de l'économie?

\section{Des activités non agricoles différenciées}

Le terme d'activité non agricole recouvre une grande diversité d'activités. Celles mises en œuvre dans les deux cantons peuvent être classées de la manière suivante, par ordre de rémunération annuelle globalement croissant :

i. salariat temporaire (journalier à quelques semaines) physiquement exigeant (manutention, coupe de bois, maçonnerie, chantiers divers, nettoyage, industrie) ;

ii. salariat permanent non qualifié (vendeur, chargé du nettoyage, gardien, serveur, etc.) ;

iii. professeur des écoles ;

iv. petit entreprenariat commercial avec auto emploi ; 
v. salariat permanent qualifié (employé de banque, des services de santé, des coopératives, des services vétérinaires, d'industries, de l'administration, directeur d'école);

vi. entreprenariat avec investissements importants (restaurant, immobilier, industrie locale, entreprise de travaux agricoles, scierie).

Ces activités non agricoles ne sont pas mises en œuvre aléatoirement au sein des populations étudiées. Comme cela a été mis en évidence par d'autres travaux (Thorat et al., 2005 ; Rawal, 2006 ; Breman, 2007b ; Abraham, 2009), il apparaît que le type d'emploi non agricole auquel accèdent les travailleurs ruraux dans les deux zones est étroitement dépendant de leur origine sociale et donc du type d'exploitation agricole dont ils sont issus. À Petlad, certains propriétaires Patel occupent un emploi salarié qualifié localement ou à l'étranger et les autres gèrent souvent diverses entreprises dans lesquelles ils ont investi. A Dharampur, les plus grands propriétaires producteurs de canne à sucre et de mangues ont généralement un emploi salarié qualifié dans le secteur industriel ou tertiaire des villes en croissance du Sud du Gujarat (Vapi, Surat, Valsad), et certains sont propriétaires de scierie. Dans les deux cantons, certaines exploitations familiales - les petits propriétaires de Petlad et les agriculteurs aux productions diversifiées de Dharampur - peuvent avoir l'un de leurs membres qui occupe un emploi permanent non qualifié, est professeur des écoles s'il a bénéficié du système des quotas, voire même exerce une petite activité commerciale, et diversifient ainsi leurs revenus. Il est néanmoins très rare qu'ils accèdent à des emplois non agricoles mieux rémunérés, du fait d'une capacité d'investissement et d'un niveau d'études insuffisants ${ }^{16}$. Enfin, pour les journaliers et les métayers de Petlad comme pour les agriculteurs en culture pluviale des montagnes de Dharampur, il est quasiment impossible de trouver des emplois autrement qu'à la journée, très physiques et mal rémunérés, et même au cours des mois agricoles creux, ils n'y parviennent généralement pas tous les jours. Le père, les fils les plus âgés et la mère dans une moindre mesure travaillent dans le secteur des services qui est en croissance dans les petites villes (cas des Adivasis à Dharampur), la construction ou les travaux publics. Pour des raisons de coût des transports et de logement, il leur est généralement difficile d'explorer des marchés du travail tout aussi physique mais plus rémunérateur comme Vapi, qui sont plus éloignés.

\section{Pour les plus pauvres, nécessité et fragilité de l'emploi non agricole}

Pour les familles les plus pauvres, les activités non agricoles correspondent à une impérieuse nécessité face à la faiblesse des revenus tirés de l'agriculture. Comme le montrent les illustrations 2 et 3, les agriculteurs en culture pluviale de Dharampur, les journaliers et une partie des métayers de Petlad ne peuvent pas vivre exclusivement de l'agriculture et c'est là un puissant moteur d'engagement dans des activités non agricoles, quelles qu'elles soient. Les revenus totaux qu'ils obtiennent en combinant activités agricoles et non agricoles, qui sont proches du seuil officiel de pauvreté (28 000 Rs par an pour les 2,5 personnes que doit faire vivre un actif), témoignent de cette fragilité : un éleveur sans terre avec une bufflesse à Petlad travaillant comme journalier dans la maçonnerie 14 jours par mois, dégage un revenu annuel total de $25000 \mathrm{Rs}$; un agriculteur en culture pluviale des montagnes de Dharampur qui parvient à être embauché 100 jours par an en dehors de son exploitation comme journalier, obtient au maximum un revenu annuel total de $35000 \mathrm{Rs}^{17}$. Ainsi, l'essor des activités non agricoles ne prouve pas que ces familles aient trouvé là un moyen 
d'améliorer durablement leur situation. Elles peuvent en effet être conduites, faute d'alternative, à accepter de très mauvaises conditions de travail et de rémunération (Breman, 2007a, Harriss-White and Gooptu, 2009).

Dans les deux cantons, ce caractère contraint des activités non agricoles des ruraux les plus pauvres est susceptible d'être renforcé par les évolutions en cours. Division des terres entre générations, dégradation de la fertilité dans les montagnes de Dharampur, raréfaction des ressources en eau, baisse de la demande en travail agricole de la part des propriétaires fonciers, accroissement du prix des intrants et hausse du coût de la vie, sont autant de facteurs de dégradation des revenus issus de l'agriculture en termes réels qui rendent les activités non agricoles impératives pour les plus pauvres.

La fragilité de cette voie de sortie de la pauvreté via l'emploi non agricole est corroborée par des analyses à l'échelle de l'Inde qui suggèrent qu'au-delà de la question du niveau de salaire, le nombre de ces emplois non agricoles est très insuffisant pour absorber la masse de travailleurs ruraux impliqués dans l'agriculture (Dorin et al., 2013). La croissance économique indienne, certes forte ( $6,8 \%$ de moyenne annuelle entre 1993 et 2013), repose surtout sur le secteur des services (67 \% du PIB en 2013) généralement moins pourvoyeur d'emplois que l'industrie, et s'appuie sur un secteur industriel à forte intensité capitalistique qui génère peu d'emplois (Kannan et Raveendran, 2009). Le diagnostic de cette croissance sans emploi (jobless growth) est à l'origine de l'instauration en 2005 d'un droit au travail des ménages les plus pauvres de 100 jours par an sur des chantiers d'intérêt public via le Mahatma Gandhi National Rural Employment Guarantee Act, qui est encore peu développé semble-t-il dans les deux cantons étudiés ${ }^{18}$.

\section{Vers un abandon de l'agriculture par les plus pauvres?}

Des travaux conduits dans d'autres régions de l'Inde mettent en avant un abandon des activités agricoles par certains des plus pauvres (Gupta, 2005 ; Jodhka, 2014). Qu'en estil dans les zones étudiées? Cela pourrait se produire pour les journaliers agricoles de Petlad si la demande en travail agricole venait à diminuer, sous l'effet du remplacement du tabac par de l'eucalyptus par exemple. Ces journaliers ont d'ailleurs déjà, pour certains, arrêté l'élevage, parce que le temps qu'ils passaient à collecter des fourrages sur les bords de chemins et de canaux réduisait leur disponibilité pour occuper des emplois journaliers agricoles et non agricoles. Mais il semble que les tribaux, même s'ils vont chercher du travail ailleurs, continuent les cultures pluviales sur leurs terres afin de disposer d'un minimum de riz et de légumineuses pour nourrir la famille, ce même s'ils accèdent aux aliments distribués à bas prix dans le cadre du Public Distribution System. Les choix qu'opèrent les familles rurales entre activités agricoles et non agricoles ne peuvent être interprétés simplement comme un mouvement général des unes vers les autres. Ces activités sont souvent combinées dans ce que les chercheurs ont maintenant coutume d'appeler les "systèmes d'activités" (Dufumier, 2006; Gasselin et al., 2012). Cette combinaison est dûment réfléchie en fonction des conditions locales et de la situation de la famille qui définissent : (i) l'importance, la régularité et la sécurité du revenu agricole auquel elle peut accéder compte tenu de l'accès à la terre, à l'eau et des modalités de répartition de la valeur ajoutée ; (ii) la rémunération, la localisation, la régularité, la sécurité des emplois non agricoles auxquels elle peut accéder ; (iii) les possibilités d'affectation de la main-d'œuvre familiale entre les 
différentes activités. Si les conditions le favorisent, l'abandon complet de l'agriculture par certaines familles au profit des activités non agricoles, localement ou via la migration, n'est pas à exclure. Les journaliers agricoles sans terre sont probablement les plus enclins à emprunter ce chemin, même si des sans-terres développant l'élevage à partir de prêts des coopératives laitières témoignent d'une dynamique inverse à Dharampur. Mais rappelons-le : ce n'est pas parce que ce chemin est "non agricole » qu'il leur permet de sortir de la pauvreté. Cela pourrait même être le contraire quand on voit le type d'activités non agricoles que mettent en œuvre les familles les plus pauvres dans les deux zones. Ainsi, à moins d'être assortie d'une création d'emplois stables et rémunérateurs dans d'autres secteurs de l'économie, la sortie "forcée » de l'agriculture des journaliers agricoles travaillant dans les champs de tabac de Petlad, si les propriétaires décident de remplacer cette culture gourmande en travail par de l'eucalyptus, serait tout sauf une solution à la pauvreté.

\section{Conclusion}

L'agriculture, dans les deux zones étudiées, ne constitue pas une activité résiduelle et rares sont les familles qui, dans les faits, l'abandonnent. Même s'ils ne les travaillent pas directement et qu'ils ne résident parfois plus en Inde, les propriétaires conservent leurs terres ; les familles qui ont très peu de terres en propriété - ou des terres sans eau - les gardent comme sécurité et, comme les sans-terres, combinent activités agricoles et non agricoles. L'agriculture contribue ainsi à faire vivre des dizaines de milliers de familles dans les deux cantons. Elle participe également à la sécurité alimentaire de l'Inde, ce qui n'est pas un mince enjeu dans un pays qui sera bientôt le plus peuplé au monde (Landy, 2008).

La recherche réalisée confirme l'extrême pauvreté qui sévit dans les zones rurales de l'Inde, dans un État qui affiche pourtant un taux de croissance élevé, y compris dans le secteur agricole. Il montre comment cette pauvreté, dans les deux zones étudiées, s'explique par une inégale répartition de la terre, de l'eau, mais aussi de la valeur ajoutée, qui s'enracine dans des relations sociales de dépendance dont l'essence a été finalement assez peu modifiée au cours des dernières décennies. Les activités non agricoles, pour essentielles qu'elles soient devenues, notamment pour les plus pauvres, ne modifient pas ces inégalités et ne permettent donc pas d'enrayer la pauvreté. À la lumière de ce travail, la perspective selon laquelle la croissance économique de l'Inde entraînerait spontanément dans son sillon les ruraux pauvres et permettrait leur ascension sociale parait peu réaliste.

Les actions publiques visant la résorption des inégalités en zone rurale apparaissent plus que jamais légitimes. Notre travail suggère que les politiques de développement agricole incluant les plus pauvres et tenant compte de leurs intérêts constituent un moyen efficace d'atteindre un tel objectif. Dans l'histoire récente des deux zones, la réforme agraire, l'accès à l'élevage laitier pour tous dans la plaine via les coopératives laitières, les subventions ou prêts pour équipements d'irrigation permettant à certaines familles adivasis de Dharampur de développer le maraîchage ont été, chacun à leur manière, des moyens d'ascension sociale pour des familles rurales pauvres. Même si elles comportent des limites qu'il convient d'analyser, il serait sans doute bon de s'en inspirer, afin de définir des politiques permettant à l'Inde de profiter pleinement des emplois et des ressources alimentaires que l'agriculture peut lui offrir. 


\section{BIBLIOGRAPHIE}

Abraham V., 2009. Employment Growth in rural India: Distress-Driven? Economic and Political Weekly, 44, p. 97-104.

Admnistration of Dharampur, 1921. Administrative history of Dharampur. Dharampur.

Agarwal B., 2005. Landmark Step to Gender Equality. The Hindu, 25 sept 2005.

Agarwal B., 1988. Who sows? Who reaps? Women and land rights in India. The Journal of Peasant Studies, 15, p. 531-581.

Appu P.S., 1997. Land reforms in India: a survey of policy, legislation and implementation. Vikas, New Delhi.

Bagchi A.K., Das P., Chattopadhyay S.K., 2005. Growth and Structural Change in the Economy of Gujarat, 1970-2000. Economic and Political Weekly, 40, p. 3039-3047.

Banerjee P., 2005. Femmes en Inde : législation et réalités. Diogène, 212, p. 107-127.

Barry E., Issoufaly H., 2006. L'agriculture en Inde du Nord, Gujarat : histoire et perspectives. Les Indes Savantes, Paris.

Breman J., 1974. Patronage and Exploitation: Changing Agrarian Relations in South Gujarat, India. University of California Press, Berkeley and Los Angeles.

Breman J., 2007a. Labour bondage in west India: from past to present. Oxford University Press, New Delhi.

Breman J., 2007b. The poverty regime in village India: half a century of work and life at the bottom of the rural economy in South Gujarat. Oxford University Press, New Delhi, Oxford.

Breman J., Guérin I., 2009. Introduction: on bondage old and new. In Breman J., Guérin I., Prakash A. (ed.), India's Unfree Workforce of Bondage Old and New. Oxford University Press, New Delhi, p. $1-17$.

Cochet H., 2015. Controverses sur l'efficacité économique des agricultures familiales : indicateurs pour une comparaison rigoureuse avec d'autres agricultures. Revue Tiers Monde, 1/2015, p. 9-25.

Cochet H., 2011. L'agriculture comparée. Quae, Versailles.

Cochet H., Devienne S., 2006. Fonctionnement et performances économiques des systèmes de production agricole : une démarche à l'échelle régionale. Cahiers agricultures, 15, p. 578-583.

Denis E., Marius-Gnanou K., 2011. Toward a better appraisal of urbanization in India. Cybergeo : European Journal of Geography, document 569

Deumari M.S., 2011. A dissertation submitted for the degree of master of library, Petlad a study, (Gujarati).

Dixit A.K., 2009. Agriculture in a High Growth state: Case of Gujarat (1960 to 2006). Economic and Political Weekly, 44, p. 64-71.

Dorin B., Hourcade J.-C., Benoit-Cattin M., 2013. A World without Farmers? The Lewis Path Revisited. CIRED Working Paper, 47, $22 \mathrm{p}$.

Dorin B., Landy F., 2002. Agriculture et alimentation de l'Inde : les vertes années (1947-2001). Éditions Quae, Versailles. 
Dufumier M., 2007. Agriculture comparée et développement agricole. Revue Tiers Monde, 2007/3, p. 611-626.

Dufumier M., 2006. Diversité des exploitations agricoles et pluriactivité des agriculteurs dans le Tiers Monde. Cahiers agricultures, 15, p. 584-588.

Dufumier M., 2004. Agricultures et paysanneries des Tiers mondes. Karthala Editions, Paris.

Gadgil M., Guha R., 1993. This fissured land: an ecological history of India. University of California Press, Berkeley and Los Angeles.

Gasselin P., Vaillant M., Bathfield B., 2012. The activity system. A position paper, in: 10th European IFSA Symposium "Producing and Reproducing Farming Systems: New Modes of Organization for the Sustainable Food Systems of Tomorrow". Aarhus.

Guérin I., Roesch M., Venkatasubramanian G., 2007. « Ne nous libérez pas ! ». Autrepart, 43, p. 121-133.

Gupta D., 2005. Whither the Indian Village: Culture and Agriculture in 'Rural' India. Economic and Political Weekly, 40, p. 751-758.

Harriss J., Jeyaranjan J., Nagaraj K., 2012. Rural Urbanism in Tamil Nadu. Notes on a "Slater Village": Gangaikondan, 1916-2012. Review of Agrarian Studies, 2, 2.

Harriss-White B., Gooptu N., 2009. Mapping India's world of unorganized labour. Socialist Register, 37, p. 89-118.

Himanshu H., 2008. Growth, employment and poverty reduction: post-reform Indian experience. LSE Asia Research Centre Working Papers, 23.

Himanshu H., Lanjouw P., Murgai R., Stern N., 2013. Nonfarm diversification, poverty, economic mobility, and income inequality: a case study in village India. Agricultural Economics, 44, p. 461-473.

Jaffrelot C., 2006. La démocratisation paradoxale du système politique. In Jaffrelot C. (ed.), L'Inde Contemporaine. De 1950 À Nos Jours. Fayard, Paris, p. 80-106.

Jodhka S.S., 2014. Emergent Ruralities. Revisiting Village Life and Agrarian Change in Haryana. Review of rural affairs, Economic and Political Weekly, 49, p. 5-17.

Kannan K.P., Raveendran G., 2009. Growth Sans Employment: A Quarter Century of Jobless Growth in India's Organised Manufacturing. Economic and Political Weekly, 44, p. 80-91.

Kumar M.D., Narayanamoorthy A., Singh O.P., Sivamohan M.V.K., Bassi N., 2014. Unraveling Gujarat's agricultural growth story. In The Water, Energy and Food Security Nexus: Lessons from India for Development. Oxon, p. 19-38.

Kundu A., Sarangi N., Das B.P., Nayyar R., Sharma A.N., 2005. Economic growth, poverty and nonfarm employment: an analysis of rural-urban inter-linkages. In Nayyar R., Sharma A. N (ed.), Rural Transformation in India: The Role of Non-Farm Sector. Institute for Human Development, p. 137-154.

Landy F., 2010. Dictionnaire de l'Inde contemporaine. Armand Colin, Paris.

Landy F., 2008. Nourrir 2,5 milliards de personnes, plus et mieux: les défis de l'Inde et la Chine. In Charvet JP (ed.), Nourrir Les Hommes. SEDES, p. 184-224.

Lehoux H., 2014. Diagnostic agraire. Valsad district, Sud du Gujarat, Inde. Mémoire de fin d'études IRC Montpellier SupAgro. 
Lucas C., 2014. Diagnostic agraire du taluka de Petlad : rétrospective, actualité et perspectives d'un bassin d'approvisionnement de la coopérative laitière AMUL. Mémoire de fin d'études IRC Montpellier SupAgro.

Marius-Gnanou K., 2008. Debt bondage, seasonal migration and alternative issues: lessons from Tamil Nadu (India). Autrepart, 46, p. 127-142.

National Dairy Development Board, 2013. Annual Report 2012. Anand.

Nayyar R., Sharma A.N., 2005. Rural transformation in India: the role of non-farm sector. Institute for Human Development.

Patel S.A., 2011. Status of adivasis/indigenous peoples land series, Gujarat. Aakar Books, New Delhi.

Pattnaik I., Shah A., 2013. Gujarat's Agricultural Growth Model: How Sustainable it is? International Journal of Agriculture and Food Science Technology, 4, p. 281-286.

Planning Commission, Government of India, 2014. Report of the expert group to review methodology for measurement of poverty. New Delhi.

Pouchepadass J., 2006. Le monde rural. In Jaffrelot C. (ed.), L'Inde Contemporaine. De L'indépendance À Nos Jours. Fayard, Paris, p. 421-458.

Ramachandran V.K., Rawal V., Swaminathan M., 2010. Socio-economic surveys of three villages in Andhra Pradesh: A study of agrarian relations. Tulika Books, New Delhi.

Randhawa M.S., 1980. Indian council of agriculture Research. New Delhi.

Rao N., 2006. Land rights, gender equality and household food security: Exploring the conceptual links in the case of India. Food Policy, 31, p. 180-193.

Rawal V., 2006. The Labour Process in Rural Haryana (India): A Field-Report from Two Villages. Journal of Agrarian Change, 6, p. 538-583.

Scholten B.A., 2010. India's White Revolution: Operation Flood, Food Aid and Development. I.B.Tauris, Londres.

Servet J.-M., 2007. Entre protection et surexploitation: l'ambiguïté de la rémunération par avance en Inde. Autrepart, 43, p. 103-119.

Shah A., 2002. Spatial Poverty Traps in Rural India: An Exploratory Analysis of the Nature of the Causes. Journal of Social and Economic Development, 4, p. 123-147.

Shah A., 2005. Poverty and Natural Resources: Understanding the Dynamics in the Context of Dry Land Regions in Western India. CPRC-IIPA Working Paper 25.

Shah C.P., Shah K.C., 1950. Charotar Sarsangrah (Kheda Zilla Mahitigranth) Partie 1, (Gujarati).

Charotar Sarvsangrah Trust vati Lokmat Prakashan, Nadiad.

Shah T., 2009. India's Ground Water Irrigation Economy: The Challenge of Balancing Livelihoods and Environment. Quarterly Journal of Central Ground Water Board, Ministry of Water Resources, 24, p. 21-37.

Shah T., Gulati A., Shreedhar G., Jain R.C., 2009. Secret of Gujarat's agrarian miracle after 2000. Economic and Political Weekly, 44, p. 45-55.

Subramanian S., 2011. The poverty line: Getting it wrong again. Economic and Political Weekly, 46, p. 37-42.

Thorat S., Sabharwal N.S., Nayyar R., Sharma A.N., 2005. Rural non-farm employment and scheduled castes: activities, education and poverty inter-linkages. In Nayyar R., Sharma A. N 
(ed.), Rural Transformation in India: The Role of Non-Farm Sector. Institute for Human Development, p. 175-192.

\section{NOTES}

1. Selon le recensement de 2011, $31 \%$ de la population indienne est urbaine, contre $42 \%$ selon Geopolis pour cette même date (Denis et Marius-Gnanou, 2011); de même, la définition du seuil de pauvreté, qui n'est pas le même en zone rurale et urbaine, est très discutée (Subramanian, 2011).

2. La durabilité de cette croissance dans le secteur agricole est toutefois questionnée (Pattnaik and Shah, 2013 ; Kumar et al., 2014).

3. Le terme anglais "servant» utilisé pour désigner les employés permanents pourrait être traduit par « domestique » et évoque une relation qui n'était pas exclusivement salariale.

4. De Adi = «le début» et vasi = «habitant de " (voir Hardiman, cité par Breman, 2007a concernant l'usage de ce terme).

5. Il ne nous a pas été possible d'aller plus loin dans la compréhension des relations de travail en vigueur dans le passé : nous ne connaissons ni l'importance relative de ces relations de travail ni les termes précis des échanges. En particulier, nous ne savons pas si la servitude pour dette ou bonded labour des Halpati que Breman analyse dans son œuvre $(1974,2007 a)$ à partir de l'étude de villages situés à moins d'une centaine de kilomètres était pratiqué également à Dharampur.

6. Depuis 2005, un amendement au Hindu Succession Act permet aux filles d'hériter de la propriété foncière au même titre que leurs frères (Agarwal, 2005). D'après nos enquêtes de terrain, la division des terres entre les fils semble néanmoins être encore la règle dans les deux cantons. Si notre article, centré sur les inégalités entre familles, n'aborde pas les inégalités dont les femmes sont victimes au sein de ces familles, c'est bien là un trait structurant des sociétés rurales indiennes, que de nombreux auteurs soulignent à partir de leurs travaux (voir par exemple : Agarwal, 1988 ; Banerjee, 2005 ; Rao, 2006 ; Ramachandran et al., 2010).

7. Il s'agit principalement d'une irrigation par puits et forages (voir Shah, 2009).

8. AMUL est la première coopérative laitière créée dans le Gujarat en 1946 à proximité du canton de Petlad. Le modèle sera ensuite repris et diffusé ailleurs en Inde par le National Dairy Development Board au cours de l'Operation Flood, ensemble de politiques mises en œuvre entre 1970 et 1996 en partie financées par la revente de l'aide alimentaire laitière (Dorin et Landy, 2002 ; Scholten, 2010). Les coopératives laitières indiennes collectent, transforment et distribuent aujourd'hui le lait de 15 millions de producteurs, qui élèvent pour la plupart de très petits troupeaux (moins de 2,5 litres de lait collectés par jour en moyenne par famille membre, d'après les données du NDDB, 2013).

9. Le métayage est une forme d'agriculture à part de fruits. Dans l'arrangement le plus courant à Petlad, le propriétaire fournit la terre et les intrants (dont le recours aux services d'entreprenariats) et le métayer la force de travail (dont le cas échéant celle des journaliers qu'il embauche pour la récolte, et qu'il doit donc payer). À la récolte le propriétaire récupère aujourd'hui selon les cultures $3 / 4$ à $7 / 8$ des produits, le reste revenant au métayer. Même si l'autonomie des métayers dans la conduite des cultures est réduite, leur situation diffère d'un salariat à part de fruits : ce sont eux en effet qui organisent le travail des cultures; ils disposent d'une certaine marge de manœuvre dans le choix de la rotation; ils obtiennent généralement le droit de cultiver des fourrages en bord de parcelle. Ce sont autant d'avantages qui les distinguent des journaliers. L'activité d'élevage des métayers comme des journaliers est conduite indépendamment des propriétaires fonciers.

10. Le revenu agricole calculé tient compte de la production agricole vendue comme de la production agricole autoconsommée, qui est pour certaines catégories d'exploitation très 
importante. Celle-ci a été valorisée au prix du marché, auquel les agriculteurs devraient acheter ce produit s'ils ne le produisaient pas. A Petlad, les revenus calculés pour les journaliers incluent la vente de force de travail agricole dans d'autres exploitations. Il ne nous a en revanche pas été possible d'évaluer les revenus issus du travail journalier agricole des Adivasis à Dharampur, qui implique des déplacements à la journée ou pour plusieurs jours entre la montagne et la plaine, et se trouve difficile à distinguer du travail journalier non agricole. Une évaluation du revenu maximum total incluant les activités non agricoles et agricoles de cette catégorie sociale est proposée dans la dernière partie de l'article.

11. Dans ces graphiques, le revenu par actif est comparé à 2,5 fois le seuil de pauvreté, considérant qu'un actif doit faire vivre 2,5 personnes.

12. La valeur ajoutée (produit brut auquel on soustrait les consommations intermédiaires) est rapportée au nombre de journées de travail nécessaire à sa création, pour chaque système de culture (Cochet, 2015).

13. Sauf cas particuliers de propriétaires louant des terres à des parents partis à l'étranger, ce reste constitue le revenu des propriétaires. En effet, les propriétaires ne paient pas d'impôt dans le système fiscal indien et, n'empruntant pas d'argent pour les cultures, n'ont pas d'intérêts à payer.

14. 15000 euros au moment de l'étude.

15. Notre travail ne fournit pas assez d'éléments pour explorer entièrement la question, mais il semble qu'ils gagnent le Gujarat en groupe, recrutés par un intermédiaire qui va les chercher dans leur État d'origine. Cela n'est pas sans rappeler des relations salariales décrites ailleurs en Inde pour le travail dans les briqueteries (Guérin et al., 2007) ou la récolte de la canne à sucre (Marius-Gnanou, 2008), dans lesquelles l'avance des salaires joue un rôle majeur.

16. L'insertion dans certains réseaux joue probablement un rôle fort dans l'accès à ces emplois non agricoles.

17. Ces hypothèses permettent d'estimer la contribution maximale des activités non agricoles au revenu total de ces catégories sociales, qui est respectivement de $35 \%$ et de $43 \%$ pour les journaliers avec bufflesse de Petlad et les agriculteurs en culture pluviale de Dharampur. Le plus souvent, les emplois journaliers occupés par ces catégories sociales, respectivement 20 jours par mois et 100 jours par an, ne se font en effet pas exclusivement dans le secteur non agricole et correspondent pour partie à de l'emploi agricole. Remarquons que ces calculs sont effectués pour un actif masculin : la contribution des activités non agricoles au revenu total des femmes de ces catégories sociales (et donc des familles prises dans leur globalité) est inférieure à ces valeurs, les femmes ayant dans les deux zones plus de difficultés que les hommes à occuper ces emplois journaliers non agricoles très physiques.

18. Nous n'en avons pas entendu parler dans au cours de nos entretiens.

\section{RÉSUMÉS}

Dans deux cantons de l'État du Gujarat, cet article s'intéresse aux systèmes agraires et à leurs relations avec la pauvreté rurale, composante essentielle du « grand écart spatial de l'Inde ». À partir d'un travail de terrain approfondi, il confirme l'extrême pauvreté qui sévit dans les campagnes indiennes, dans un État qui affiche pourtant un taux de croissance élevé. Il montre comment cette pauvreté s'explique par une inégale répartition de la terre, de l'eau, mais aussi de la valeur ajoutée, qui s'enracine dans des relations sociales de dépendance dont l'essence a été 
peu modifiée au cours des dernières décennies. Les activités non agricoles, pour importantes qu'elles soient, ne modifient pas ces inégalités. À la lumière de ce travail, les politiques de développement agricole visant la résorption des inégalités en zone rurale apparaissent plus que jamais nécessaires.

INDEX

Mots-clés : Inde, agriculture, système agraire, zone rurale, pauvreté, inégalité

Thèmes : Sur le Champ - Sur le Terrain

\section{AUTEURS}

\section{CLAIRE AUBRON}

Claire Aubron, aubron@supagro.fr, est Enseignant chercheur à Montpellier SupAgro, UMR 868 SELMET. Chercheuse invitée à l'International Livestock Research Institute, New Delhi, Inde, elle est associée au Centre de Sciences Humaines de New Delhi.

\section{HUGO LEHOUX}

Hugo Lehoux, hugo.lehoux@gmail.com, est un ancien étudiant à l'IRC Montpellier SupAgro, stage de fin d'études CIRAD, UMR 868 SELMET, Montpellier. Ingénieur agronome, il est créateur et concepteur de l'Atlas Numérique des Agricultures du Monde http://agricultures-du-monde.com

\section{CORENTIN LUCAS}

Corentin Lucas, lucas.corentin@free.fr, est un ancien étudiant à l'IRC Montpellier SupAgro, stage de fin d'études CIRAD, UMR 868 SELMET, Montpellier. 